\title{
Variação espaço-temporal de parâmetros biofísicos e da evapotranspiração em plantios de eucalipto
}

\author{
Spatial-temporal variation of biophysical parameters and evapotranspiration in \\ eucalyptus plantations
}

\author{
Marlus Sabino' \\ Luana Bouviéll \\ Anísio Alfredo da Silva Junior ${ }^{\text {III }}$ \\ Nadja Gomes Machado'v \\ Marcelo Sacardi Biudes $\vee$
}

\section{Resumo}

Avaliou-se a variação espaço-temporal dos parâmetros biofísicos em plantios de Eucalipto, na transição CerradoAmazônia Mato-Grossense, por meio de sensoriamento remoto. A área localiza-se no município de Lucas do Rio Verde-MT, sendo composta por talhões de E. urophylla, E. urograndis, mata nativa e solo exposto. As imagens foram adquiridas no site do U. S. Geological Survey referentes aos meses de fevereiro 2015/16 e janeiro 2017 (estação chuvosa) e junho 2015-2017 (estação seca). O Índice de Vegetação da Diferença Normalizada (NDVI), albedo da superfície ( $\alpha$ ); temperatura da superfície (Ts); Saldo de Radiação (Rn) e evapotranspiração (ET) foram calculados em etapas do modelo R-SSEB. Os plantios de Eucalipto, comparado a floresta nativa, apresentaram aumento de 3\% a 4\% nos valores de NDVI, Ts, Rn e ET. A exposição de solo provocou aumento do albedo (69\% a 109\%) e da Ts (14\% a 34\%) e redução do NDVI (63\% a 58\%), do Rn (13\% a 20\%) e da ET (57\% a 26\%), nos períodos de chuva e seca, respectivamente. Conclui-se que as mudanças no uso e ocupação do solo alteraram os parâmetros biofísicos e a evapotranspiração, sendo estas mais acentuadas em áreas desmatadas do que em áreas com cultivo de Eucalipto.

Palavras-chave: mudança do uso do solo; desmatamento; necessidade hídrica; microclima

\section{Abstract}

The spatiotemporal variation of biophysical parameters in Eucalipto plantations, in the Savanna-Amazonian MatoGrossense transition, was evaluated by means of remote sensing. The area is located in the municipality of Lucas do Rio Verde-MT, and it is composed of plots of E. urophylla, E. urograndis, native forest and exposed soil. The images were acquired on the website of the U. S. Geological Survey for the months of February 2015/16 and January 2017 (rainy season) and June 2015-2017 (dry season). The Normalized Difference Vegetation Index (NDVI), surface albedo ( $\alpha$ ); surface temperature (Ts); Radiation Balance $(\mathrm{Rn})$ and evapotranspiration (ET) were calculated in steps of the R-SSEB model. Eucalyptus plantations, compared to native forest, showed an increase of 3\% to 4\% in NDVI, Ts, Rn and ET values. Soil exposure increased albedo (69\% to $109 \%)$ and Ts (14\% to 34\%) and reduced NDVI (63\% to 58\%), $\mathrm{Rn}(13 \%$ to $20 \%)$ and ET (57\% to $26 \%)$, in the rainy and dry periods, respectively. It is concluded that the changes in soil use and occupation altered the biophysical parameters and evapotranspiration, being these more accentuated in deforested areas than in areas with Eucalyptus cultivation.

Keywords: land use change; deforestation; water requirement; microclimate

' Universidade Federal do Mato Grosso, MT, Brasil - marlussabino@gmail.com

"Universidade Federal do Mato Grosso, MT, Brasil - bouvieluana@gmail.com

"' Universidade Federal do Mato Grosso, MT, Brasil - anisio.silva@fisica.ufmt.br

Iv Instituto Federal do Mato Grosso, MT, Brasil - nadja.machado@blv.ifmt.edu.br

v Universidade Federal do Mato Grosso, MT, Brasil - marcelo@fisica.ufmt.br 


\section{Introdução}

As florestas tropicais são ecossistemas que desempenham papel chave na regulação do clima em escala local e regional. O Brasil se destaca por possuir a maior cobertura de florestas tropicais do mundo associado à megadiversidade biológica e heterogeneidade fisiográfica e climática de seu território (LEWINSOHN; PRADO, 2000). Essa heterogeneidade é evidenciada em Estados como o Mato Grosso, que abriga três biomas: a Floresta Tropical Úmida (Amazônia), o Cerrado e a planície do Pantanal, com regiões de transição entre eles (TANAKA et al., 2016).

A vegetação nativa desses biomas é importante por atuar no fornecimento de vapor de água para a atmosfera, pela evapotranspiração, que influenciam na circulação das massas de ar e na formação das chuvas (ARTAXO et al., 2014; DOUGHTY et al., 2015). Contudo, apesar dos serviços ambientais desempenhados por esses ecossistemas, parte da cobertura natural do Mato Grosso tem sido removida em consequência da expansão da agropecuária e do crescimento populacional nas últimas décadas (DOMINGUES et al., 2012).

Áreas degradadas na região de transição Cerrado-Amazônia estão sendo utilizadas para produção de eucalipto a fim de suprir a demanda de madeira para fins energéticos das indústrias e secadores no Mato Grosso. Essa espécie exótica vem sendo utilizada devido a adaptabilidade às condições edafoclimáticas do Estado e ser a principal cultivo energético do país, representando, em 2012 , cerva de $72 \%$ da área total de florestas plantadas no Brasil (ABRAF, 2013; FAMATO, 2013).

A substituição das florestas nativas por monoculturas, contudo, modificam o microclima, podendo causar alterações na temperatura do ar, no albedo da superfície, na partição da energia disponível no sistema e, consequentemente, na evapotranspiração (SALAZAR et al., 2015; VOURLITIS et al., 2015). Assim, o monitoramento de variáveis micrometeorológicas, incluindo evapotranspiração, é importante na quantificação dos impactos antrópicos nos ecossistemas em escala espacial e temporal.

Variáveis microclimáticas são obtidas, em geral, de forma pontual a partir de dados de superfície devido ao elevado custo e dificuldade de manutenção dos equipamentos (SCHUURMANS, 2003). Assim, o desenvolvimento da tecnologia de satélites ambientais possibilitou a obtenção de informações em grandes áreas com grande facilidade e operacionalidade, por meio de modelos matemáticos com precisão e acurácia aceitáveis (ARAUJO et al., 2017).

Alguns modelos ganharam popularidade nas últimas duas décadas, tal qual, o NDVI - Normalizaed Difference Vegetation Index, proposto por Rouse et al. (1974), que funciona como um indicador de crescimento e vigor da vegetação e podem ser correlacionados com a biomassa, porcentagem de cobertura do solo, atividade fotossintética e com a produtividade do ecossistema em estudo (PONZONI, 2001) e o Simplified Modeling for Energy Balance Estimation to the Regional Scale Surface (R-SSEB), que possibilita o cálculo da evapotranspiração por meio de uma relação entre o albedo, a temperatura da superfície e o NDVI (ARAUJO et al., 2017).
Diante da importância econômica da produção de madeira de eucalipto e do potencial impacto no clima regional pela substituição da vegetação nativa, o objetivo desse estudo foi avaliar a variação espaço-temporal da de parâmetros biofísicos e da evapotranspiração da superfície em uma área de plantio de eucalipto de diferentes idades e de um fragmento de floresta nativa na região de transição Cerrado-Amazônia no estado de Mato Grosso.

\section{Materiais e Métodos}

\section{1. Área de estudo}

A área de estudo está localizada em uma fazenda no município de Lucas do Rio Verde, microrregião de Alto Teles Pires e mesorregião do Norte Mato-grossense. O clima da região é Aw segundo Köppen, temperatura média anual de $25^{\circ} \mathrm{C}$ e precipitação média anual de $2.300 \mathrm{~mm}$. A vegetação é constituída por Cerrado stricto sensu, Cerrado arbóreo denso (cerradão) e matas ciliares. O relevo é plano com predomínio de solos argilosos, areno-argilosos e latossolos vermelho amarelo distrófico (FERREIRA, 2001).

A fazenda possui uma área de 478 ha destinada ao plantio de Eucalyptus urophylla GG100 e Eucalyptus urograndis H13 para geração de energia. Foram avaliados 4 talhões de Eucalipto, plantados em espaçamento $2 \mathrm{~m}$ x 1,5 m, com diferentes idades. Os talhões 2 ( $24 \mathrm{ha})$ e 4 ( $28 \mathrm{ha})$ foram plantados como a espécie E. urograndis no início de 2013 e 2017, respectivamente. Os talhões 3 (24 ha) e 5 (12 ha) foram plantados com E. urophylla nos anos de 2007 e 2009. Contudo, o talhão 3 foi colhido no período de estiagem de 2016. Além dos talhões de Eucalipto foi utilizada uma área nativa (talhão 1 - 109 ha) adjacente à fazenda como tratamento testemunha (Figura 1).

\subsection{Aquisição e processamento das imagens}

Foram utilizadas 6 imagens de refletância da superfície e temperatura da superfície do Operational Land Imager (OLI) and Thermal Infrared Sensor (TIRS), geradas a bordo do satélite Landsat 8, na orbita 227 e ponto 69, obtidas junto à U. S. Geological Survey (espa.cr.usgs.gov), com resolução espacial de $30 \mathrm{~m}$ x $30 \mathrm{~m}$, e correspondentes aos períodos de chuva e estiagem dos anos de 2015 a 2017 (Tabela 1). A imagem da refletância da superfície passou por correção radiométrica, correção atmosférica, correção geométrica sistemática, correção de precisão usando chips de controle de chão e o uso de um modelo digital de elevação para corrigir erro de paralaxe devido ao relevo topográfico local (CLAVERIE et al., 2015).

\subsection{Estimativa dos parâmetros biofísicos e Evapotranspiração (ET)}

A estimativa de evapotranspiração utilizada no estudo foi obtida como parte do Algoritmo R-SSEB (Simplified Modeling for Energy Balance Estimation to the Regional Scale Surface) (ARAÚJO, 2017). A vantagem do algoritmo R-SSEB consiste em uma metodologia simples para a de- 
Figura 1 - Localização e delimitação dos Talhões da área de estudo em Lucas do Rio Verde - MT, 2016.

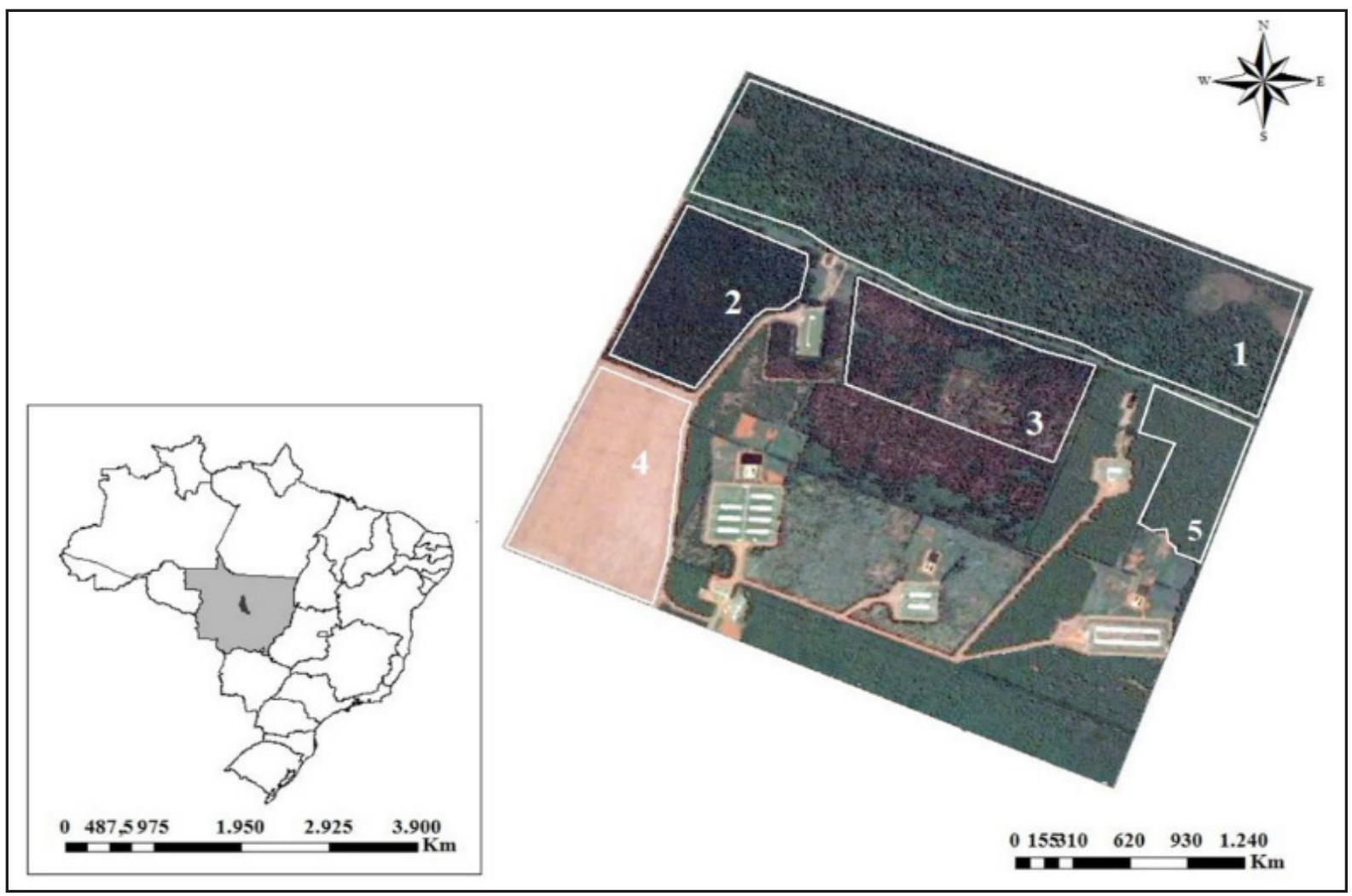

Tabela 1 - Data, horário, cosseno ângulo zenital (Cos Z), obtidos nos metadados das imagens utilizadas, valores de radiação solar instantânea (Rg), temperatura do ar (Tar) e umidade relativa do ar (UR) no momento da passagem do Landsat 8 na órbita 227 e ponto 69, bem como radiação solar diária (Rg), registrados pela estação automática do Instituto Nacional de Meteorologia - INMET/ Sorriso - MT.

\begin{tabular}{rcccccc}
\hline Data - Hora & Cos Z & $\begin{array}{c}\text { UR } \\
(\%)\end{array}$ & $\begin{array}{c}\text { Tar } \\
\left({ }^{\circ} \mathrm{C}\right)\end{array}$ & $\begin{array}{c}\text { Ea } \\
(\mathrm{kPa})\end{array}$ & $\begin{array}{c}\mathrm{Rg}\left(\mathrm{W} \mathrm{m}^{-2}\right) \\
\text { Instantâneo }\end{array}$ & Diário \\
\hline 03/02/2015-13:50 & 0,8539 & 60,00 & 29,9 & 2,53 & 840,5 & 264,0 \\
27/06/2015-13:51 & 0,6879 & 48,00 & 28,8 & 1,90 & 649,6 & 205,0 \\
06/02/2016-13:51 & 0,8537 & 68,00 & 28,3 & 2,62 & 448,1 & 204,0 \\
$13 / 06 / 2016-13: 50$ & 0,6947 & 36,00 & 26,6 & 1,25 & 330,9 & 211,0 \\
$07 / 01 / 2017-13: 51$ & 0,8633 & 65,00 & 30,3 & 2,81 & 762,3 & 229,0 \\
$16 / 06 / 2017-13: 50$ & 0,6925 & 39,00 & 31,4 & 1,79 & 610,2 & 205,0 \\
\hline
\end{tabular}

terminação do saldo de radiação e, por consequência, da ET, com base apenas no contraste da fração evaporativa entre áreas secas e úmidas.

O saldo de radiação diário $\left(R n_{24}\right)$, representa a contabilização dos fluxos radiativos à superfície em 24 horas, pode ser obtido através do cômputo dos fluxos ascendentes e descendentes das radiações de onda longa e curta atuantes no sistema solo-planta-atmosfera, Equação 1.

$$
R n_{24}=R_{S \downarrow}\left(1-\alpha_{\text {sup }}\right)-R_{L \uparrow}+R_{L \downarrow}-\left(1-\varepsilon_{0}\right) R_{L \downarrow}
$$

em que $R_{S} \downarrow$ é a radiação de ondas curtas incidente $\left(\mathrm{Wm}^{-2}\right)$, é o albedo da superfície (adimensional), $R_{L} \downarrow$ é a radiação de ondas longas emitidas pela atmosfera na direção de da superfície $\left(\mathrm{Wm}^{-2}\right), R_{L \uparrow}$ é a radiação de ondas longas emitidas pela superfície; e é a emissividade.

Visto que a radiação solar de onda curta incidente $\left(R_{S} \downarrow\right)$ é a principal fonte de energia para a ET, a Equação 1 pode ser simplificada conforme Equação 2, na qual, a radiação global representa a soma da radiação de onda curta, direta e difusa, que alcança a superfície da Terra $\left(\mathrm{W} \mathrm{m} \mathrm{m}^{-2}\right.$ ), sendo atenuada, juntamente com os componentes de onda longa, pelas propriedades da transmissividade atmosférica e albedo da superfície.

$$
R n_{24}=R g_{24} \times\left(1-\alpha_{\text {sup }}\right)-\left(110 \times \tau_{24}\right)
$$


em que $R g_{24}$ é a radiação global diária $\left(\mathrm{W} \mathrm{m}^{-2}\right)$ obtida de uma estação meteorológica automática integrante da rede do INMET próxima a área de estudo; $\alpha_{\text {sup }}$ é o albedo da superfície; e é a transmissividade média diária da atmosfera.

O albedo da superfície ( $\alpha_{\text {sup }}$ ) foi calculado de acordo com a Equação 3, proposta por Tasumi et al., (2008).

$$
\alpha_{\text {sup }}=\frac{\alpha_{t o a}-\alpha_{a t m}}{\tau_{s w}^{2}}
$$

em que é a porção da radiação solar refletida pela atmosfera, adotou-se 0,03 de acordo com Bastiaanssen (2000); é a transmitância atmosférica; e representa o albedo da superfície, sem correção atmosférica.

O albedo da superfície, sem correção atmosférica, foi obtido através de combinação linear das refletâncias espectrais das bandas ( $\rho$ ) 2, 3, 4, 5, 6 e 7, Equação 4. A transmitância atmosférica para dias de céu claro, Equação 5 , foi determinada conforme proposto por Allen et al. (2007).

$$
\begin{gathered}
\alpha_{\text {toa }}=0,300_{\rho 2}+0,277_{\rho 3}+0,233_{\rho 4}+0,143_{\rho 5}+0,036_{\rho 6}+0,012_{\rho 7} \\
\tau_{s w}=0,35+0,627 \exp \left[\frac{-0,00146 P}{K_{t} \cos \theta_{\text {hor }}}-0,075\left(\frac{W}{\cos \theta_{\text {hor }}}\right)^{0,4}\right]
\end{gathered}
$$

em que $P$ é a pressão atmosférica $\mathrm{kPa} ; W$ é água precipitável na atmosfera; $\cos \theta_{\text {hor }}$ é ângulo zenital solar sobre uma superfície horizontal; $\mathrm{K} t$ é o coeficiente de turbidez $(K \mathrm{t}=1,0$ para ar limpo e $K t=0,5$ para ar extremamente turvo, empoeirado ou poluído).

O Índice de Vegetação por Diferença Normalizada (NDVI) que serve como medida de avaliação da condição da vegetação é dado pela Equação 6, conforme proposto por Huete et al. (2002).

$$
N D V I=\frac{\rho_{I V P}-\rho_{V E R}}{\rho_{I V P}+\rho_{V E R}}
$$

em que $\rho_{I V P}$ é a refletância do infravermelho próximo; e $\rho_{V E R}$ é a refletância do vermelho.

No modelo R-SSEB a fração evaporativa (FE) é calculada utilizando a média de pelo menos três pixels quentes e três pixels frios identificados por meio de cartas de NDVI e Ts (SENAY et al., 2007). Partindo do pressuposto de que os pixels quentes apresentam valores pequenos de ET e os pixels frios representam a ET máxima ao longo de toda área de estudo, a média de cada conjunto de pixels pode ser utilizada para calcular a FE para todos os pixels da cena a partir da Equação 7 desenvolvida por Senay et al. (2013). Por fim, a evapotranspiração diária $\left(E T_{24}\right)$ é obtida conforme Equação 8.

$$
\begin{aligned}
& F E=\frac{T_{H}-T_{S}}{T_{H}-T_{C}} \\
& \mathrm{ET}_{24}=\frac{\left(\mathrm{FE}_{24} \times \mathrm{Rn}_{24} \times 0,864\right)}{\mathrm{L}}
\end{aligned}
$$

em que $T_{H}$ é a média dos pixels quentes, $T_{C}$ é a média dos pixels frios, $T_{S}$ é a temperatura da superfície, $E T_{24}$ é a evapotranspiração diária $\left(\mathrm{mm} \mathrm{dia}^{-1}\right) ; R n_{24}$ é o saldo de radiação diário $\left(\mathrm{W} \mathrm{m}^{-2} \mathrm{dia}^{-1}\right) ; L$ é calor latente de vaporização da água $\left(2,451 \mathrm{KJ} \mathrm{kg}^{-1}\right)$; e $F E_{24}$ é a fração evaporativa diária.

\subsection{Análise estatística}

Após o cálculo das estimativas dos parâmetros biofísicos (NDVI, albedo, temperatura, saldo de radiação) e da evapotranspiração da superfície foram selecionados de forma aleatória, em cada imagem (período), 100 pixels por tratamento (talhão), totalizando 3000 pixels. Os dados biofísicos e de evapotranspiração extraídos dos pixels foram submetidas ao teste de normalidade de Kolmogorov Smirnov e, após verificada a não normalidade da distribuição, as médias das variáveis foram comparadas pelo teste não paramétrico de Kruskal-Wallis a $0,05 \%$ de significância, usando o Package PMCMR do software R. Foram ainda geradas figuras dos parâmetros biofísicos e da evapotranspiração na área de estudos para os 6 períodos avaliados, sendo determinado o número de intervalos de classes da distribuição dos dados dos parâmetros pela regra de Sturges.

\section{Resultados e Discussão}

O NDVI da área de floresta de transição variou entre 0,80 e 0,88 , semelhantes aos encontrados em ambas as espécies de Eucalipto com mais de 2 anos de idade (talhões 02 e 05), que variou entre 0,81 e 0,90. Na área de Eucalipto com até 1 ano de idade (Talhão 04 - 2017), contudo, observou-se aumento do NDVI $(0,92$ a 0,94$)$, indicando maior atividade fotossintética das plantas no estágio inicial. Em contrapartida, a conversão de áreas de floresta em solo exposto foi responsável pela redução de aproximadamente 66\% no NDVI (Tabela 2; Figura 2).

Apesar do NDVI ter sido semelhante entre a floresta de transição e o plantio de Eucalipto, os valores do NDVI tenderam a ser menores na área nativa, corroborando com os resultados encontrados por Silva et al. (2015) em uma área de plantio de Eucalipto em regiões de remanescentes de Cerrado no estado de São Paulo. Esse resultado indica maior atividade fotossintética das espécies de Eucalipto em relação as espécies nativas do Cerrado. Valores de NDVI superiores a 0,70 , contudo, são comuns em floresta e indicam uma vegetação sadia em pleno crescimento (PAVÃO et al., 2016). É comum ainda obter valores entre 0,80 e 0,90 em diversas espécies vegetais como demonstrado por Cristiano et al. (2014) ao comparar o NDVI de um fragmento de Mata Atlântica na Argentina com plantios comerciais de Eucalyptus grandis, Araucaria angustifolia e Pinus taeda.

O NDVI do Eucalipto apresentou maior homogeneidade espacial por se tratar de um povoamento clonal (Figura 2), o que tende a ter maior densidade e uniformidade de cobertura de dossel (PONZONI et al., 2015; GODOY GOERGEN et al., 2016). O NDVI em diversos ecossistemas varia sazonalmente (CRISTIANO et al., 2014), podendo, durante o período de estiagem, reduzir de 25 a 50\% em florestas semidecíduas e decíduas no Cerrado (OLIVEIRA et al., 2012) devido a perca de parte ou toda a área foliar. Maiores valores de NDVI na estação chuvosa 
Tabela 2. Médias do Índice de Vegetação da Diferença Normalizada (NDVI), do albedo da superfície, da temperatura de brilho da superfície $\left({ }^{\circ} \mathrm{C}\right)$, do saldo de radiação diária (Wm-2 dia-1) e da evapotranspiração diária (mm dia-1) em diferentes uso e ocupação do solo da área de estudo entre os anos de 2015 a 2017.

\begin{tabular}{|c|c|c|c|c|c|c|}
\hline \multirow{3}{*}{ Talhões (Plantio) * } & \multicolumn{2}{|c|}{2015} & \multicolumn{2}{|c|}{2016} & \multicolumn{2}{|c|}{2017} \\
\hline & Chuva & Seca & Chuva & Seca & Chuva & Seca \\
\hline & \multicolumn{6}{|c|}{ NDVI } \\
\hline 1- Mata Nativa & $0,85 \mathrm{Bb}$ & $0,80 \mathrm{ABC}$ & $0,88 \mathrm{ABa}$ & $0,84 \mathrm{Bbc}$ & $0,85 \mathrm{Bb}$ & $0,82 \mathrm{Cbc}$ \\
\hline 2 - E. urograndis (2013) & $0,89 \mathrm{Aa}$ & $0,81 \mathrm{ABC}$ & $0,90 \mathrm{Aa}$ & $0,86 \mathrm{Bb}$ & 0,83 Cc & $0,84 \mathrm{Cbc}$ \\
\hline 3 - E. urophylla (2007) ** & $0,86 \mathrm{Ba}$ & $0,79 \mathrm{Bb}$ & $0,84 \mathrm{Ca}$ & $0,28 \mathrm{Cc}$ & 0,29 Dc & $0,75 \mathrm{Db}$ \\
\hline 4- E. urograndis (2017) & $0,75 \mathrm{Cb}$ & $0,42 \mathrm{Cc}$ & 0,33 Dc & $0,35 \mathrm{Cc}$ & $0,94 \mathrm{Aa}$ & $0,92 \mathrm{Aa}$ \\
\hline \multirow[t]{2}{*}{5 - E. urophylla (2009) } & $0,88 \mathrm{Aa}$ & $0,84 \mathrm{Ab}$ & $0,87 \mathrm{Ba}$ & $0,88 \mathrm{Aa}$ & $0,87 \mathrm{Ba}$ & $0,87 \mathrm{Ba}$ \\
\hline & \multicolumn{6}{|c|}{ Albedo } \\
\hline 1- Mata Nativa & $0,12 \mathrm{Db}$ & $0,12 \mathrm{Cb}$ & $0,14 \mathrm{Ca}$ & $0,12 \mathrm{Cb}$ & $0,13 \mathrm{Cb}$ & $0,12 \mathrm{Cb}$ \\
\hline 2 - E. urograndis (2013) & $0,14 \mathrm{CDb}$ & $0,17 \mathrm{Ba}$ & $0,17 \mathrm{Ba}$ & $0,14 \mathrm{Cb}$ & $0,15 \mathrm{Cb}$ & $0,15 \mathrm{Cb}$ \\
\hline 3 - E. urophylla $(2007)^{* *}$ & $0,15 \mathrm{BCd}$ & $0,17 \mathrm{Bc}$ & $0,16 \mathrm{Bc}$ & $0,27 \mathrm{Aa}$ & $0,26 \mathrm{Aab}$ & $0,20 \mathrm{Ab}$ \\
\hline 4 - E. urograndis (2017) & $0,18 \mathrm{Ab}$ & $0,26 \mathrm{Aa}$ & $0,22 \mathrm{Aab}$ & $0,23 \mathrm{Aab}$ & $0,19 \mathrm{Aab}$ & $0,16 \mathrm{Bc}$ \\
\hline \multirow[t]{2}{*}{5 - E. urophylla (2009) } & $0,16 \mathrm{Bb}$ & $0,17 \mathrm{Ba}$ & $0,19 \mathrm{Aa}$ & $0,16 \mathrm{Bb}$ & $0,17 \mathrm{Ba}$ & $0,16 \mathrm{Bb}$ \\
\hline & \multicolumn{6}{|c|}{ Temperatura da superfície $\left({ }^{\circ} \mathrm{C}\right)$} \\
\hline 1- Mata Nativa & $21,1 \mathrm{Bcd}$ & 23,3 Ba & $20,5 \mathrm{Bd}$ & $21,7 \mathrm{Bbc}$ & $22,1 \mathrm{Bb}$ & $25,2 \mathrm{Ba}$ \\
\hline 2 - E. urograndis (2013) & $21,2 \mathrm{Bd}$ & $24,3 \mathrm{Bb}$ & $20,5 \mathrm{Bd}$ & $22,3 \mathrm{BC}$ & $22,6 \mathrm{BC}$ & $26,2 \mathrm{Aa}$ \\
\hline $3-$ E. urophylla (2007) $)^{* *}$ & $21,8 \mathrm{Bd}$ & $24,3 \mathrm{BC}$ & $20,6 \mathrm{Be}$ & $29,8 \mathrm{Aa}$ & $25,8 \mathrm{Abc}$ & $27,5 \mathrm{Aab}$ \\
\hline 4 - E. urograndis (2017) & $22,8 \mathrm{Abc}$ & 28,0 Aa & 23,3 Ab & $29,1 \mathrm{Aa}$ & $21,9 \mathrm{BC}$ & 25,9 Aa \\
\hline \multirow[t]{2}{*}{5 - E. urophylla (2009) } & $21,5 \mathrm{Bb}$ & $23,8 \mathrm{Ba}$ & $20,4 \mathrm{Bd}$ & $21,9 \mathrm{Bbc}$ & $21,3 \mathrm{Bc}$ & 25,9 Aa \\
\hline & \multicolumn{6}{|c|}{ Saldo de Radiação (W m-2 dia $^{-1}$ ) } \\
\hline 1- Mata Nativa & $168,5 \mathrm{Aa}$ & $113,1 \mathrm{Ac}$ & $125,6 \mathrm{Abc}$ & $118,4 \mathrm{Ac}$ & $143,3 \mathrm{Ab}$ & $114,1 \mathrm{Ac}$ \\
\hline 2 - E. urograndis (2013) & $164,8 \mathrm{Aa}$ & $105,4 \mathrm{Bd}$ & $121,4 \mathrm{Ac}$ & $115,4 \mathrm{ABC}$ & $140,7 \mathrm{Ab}$ & $108,4 \mathrm{ABd}$ \\
\hline 3 - E. urophylla (2007) ** & $162,9 \mathrm{Aa}$ & $105,7 \mathrm{Bc}$ & $122,6 \mathrm{Ab}$ & $85,1 \mathrm{Cd}$ & $113,7 \mathrm{Bb}$ & $99,3 \mathrm{Ccd}$ \\
\hline 4 - E. urograndis (2017) & $151,1 \mathrm{Aa}$ & $87,3 \mathrm{Cc}$ & $108,5 \mathrm{Bb}$ & $95,0 \mathrm{Cc}$ & $131,5 \mathrm{Aa}$ & $105,6 \mathrm{BCb}$ \\
\hline \multirow[t]{2}{*}{5 - E. urophylla (2009) } & $159,4 \mathrm{Aa}$ & $103,1 \mathrm{BCC}$ & $117,2 \mathrm{Ab}$ & $108,9 \mathrm{BC}$ & $136,3 \mathrm{Aa}$ & $104,4 \mathrm{BCd}$ \\
\hline & \multicolumn{6}{|c|}{ Evapotranspiração (mm dia $\left.{ }^{-1}\right)$} \\
\hline 1- Mata Nativa & $4,9 \mathrm{Aa}$ & 3,3 Ac & $4,2 \mathrm{Ab}$ & 3,9 Abc & $5,2 \mathrm{Aa}$ & $3,4 \mathrm{Ac}$ \\
\hline 2 - E. urograndis (2013) & 4,6 ABa & $2,6 \mathrm{Bc}$ & $3,9 \mathrm{Ab}$ & 3,5 Ab & $4,7 \mathrm{Aa}$ & $2,8 \mathrm{Ac}$ \\
\hline 3 - E. urophylla (2007) ${ }^{* *}$ & 4,2 Ba & $2,7 \mathrm{Bb}$ & $4,0 \mathrm{Aa}$ & $0,7 \mathrm{BC}$ & 2,3 Bbc & $2,1 \mathrm{Bbc}$ \\
\hline 4 - E. urograndis (2017) & $3,4 \mathrm{Cb}$ & $1,0 \mathrm{Cc}$ & $2,4 \mathrm{BC}$ & $1,0 \mathrm{Bc}$ & $4,6 \mathrm{Aa}$ & $2,8 \mathrm{Ab}$ \\
\hline 5 - E. urophylla (2009) & 4,5 ABab & $2,8 \mathrm{Bd}$ & $4,0 \mathrm{Abc}$ & $3,5 \mathrm{Acd}$ & 4,8 $\mathrm{Aa}$ & $2,9 \mathrm{Ad}$ \\
\hline
\end{tabular}

Médias seguidas de letras iguais, maiúsculas na coluna e minúscula na linha, não diferem estatisticamente pelo método de Kruskal-Wallis a 0,05\% de significância. ${ }^{*}$ Plantio ocorreram no início de cada ano (Período Chuvoso) ${ }^{* *}$ Colhido no período seco de 2016. 
Figura 2 - Índice de Vegetação da Diferença Normalizada (NDVI) em diferentes usos do solo, nos períodos sazonais de 2015 a 2017.

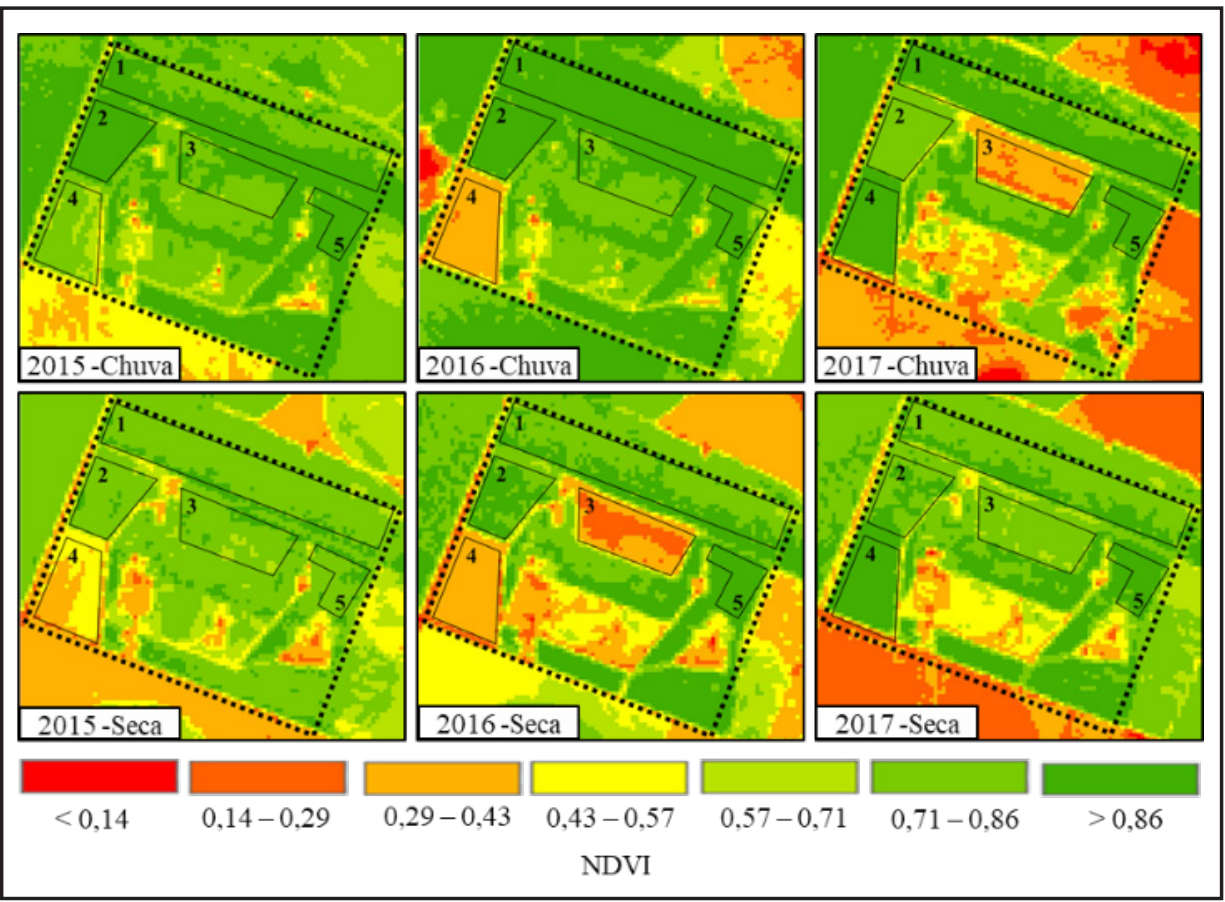

são justificando, em geral, pela maior disponibilidade de água na superfície, o que aumenta a capacidade fotossintética da vegetação (CUNHA et al., 2012; MARTINS et al., 2015), e menores valores de NDVI estão relacionados com estresse hídrico (FU; BURGHER, 2015).

O NDVI foi $4,6 \%$ menor durante o período de estiagem na área de floresta nativa e $3,4 \%$ nos talhões de Eucalipto, o que sugere que a redução da disponibilidade hídrica em meados de junho, correspondente a metade do período de seca, não é forte o suficiente para limitar o desenvolvimento das plantas. Esse mesmo padrão foi observado por Alvares et al. (2013), em plantações de Pinus no estado de São Paulo, onde houve pouca variação do NDVI no início do período de deficiência hídrica, por outro lado, o NDVI diminuiu repentinamente em torno de $20 \%$ no final da estação seca devido ao maior estresse hídrico.

O balanço hídrico do estado do Mato Grosso, realizado por Souza et al. (2013), demonstra que na região da área de estudo, o início do período de deficiência hídrica ocorre em abril e o máximo entre agosto e setembro. Contudo, em junho ainda existe retirada de água do solo o que justificaria as pequenas reduções no NDVI na floresta nativa e nos talhões de Eucalipto. Além disso, tanto a diversas espécies de árvores da floresta de transição Cerrado-Amazônia quanto o Eucalipto apresentam mecanismos de exploração de água em camadas profundas do solo, como o crescimento de raízes com até $20 \mathrm{~m}$ de profundidade, que permitem o acesso a grandes quantidades de água armazenada durante a estação seca (FISHER et al., 2007; BRANNSTROM et al., 2008; LACLAU et al. 2013; CHRISTINA et al., 2018).

Pequenas reduções do NDVI foram relatadas em áreas experimentais em que não ocorrem déficit hídrico ao longo do ano, conforme observado por Cristiano et al. (2014) que encontrou reduções no NDVI de $2,2 \%$ em $E$. grandis e 1,9\% em Mata Atlântica, assim como, Alvares et al. (2013) e Trentin et al. (2018) que encontraram, no estado do Rio Grande do Sul, variações intra-anuais menores que $10 \%$ em plantios de Pinus e Eucalipto, respectivamente, sendo essas variações atribuídas a sazonalidade da temperatura do ar. Mesmo em condições em que a disponibilidade hídrica não é o fator limitante ao desenvolvimento vegetal, temperaturas elevadas podem causar fechamento estomático, prejudicar a atividade enzimática e diminuir a eficiência do sistema de captação de luz e, consequentemente, diminuir a assimilação de $\mathrm{CO}_{2}$ e o crescimento de plantas (SHARKEY, 2005; LLOYD; FARQUHAR, 2008).

Os menores valores de albedo foram observados na mata nativa $(0,12$ a 0,14$)$. Esses resultados foram semelhantes aos obtidos em regiões de Floresta Amazônica e de Cerrado, o que demonstra que o albedo em áreas de floresta densa tende a ser próximo a 0,11 (ANDRADE et al. 2010; PAVÃO et al. 2014; PAVÃO et al. 2015) e próximo de 0,13 em áreas de Cerrado (PEREIRA et al. 2007). O albedo no Eucalipto variou entre 0,14 e 0,19 , tendendo a ser $18 \%$ a $25 \%$ maior que em área de floresta nativa (Tabela $2 \mathrm{e}$ Figura 3(a)). O maior valor de albedo foi encontrado no talhão $4(0,26)$, nos períodos anteriores a chuva de 2017 , e talhão $3(0,27)$, nos anos posteriores a estiagem de 2016, devido a exposição do solo nas etapas de pré-plantio e pós colheita do Eucalipto, respectivamente.

A temperatura da superfície na área de floresta nativa variou entre 20,5 e $25,2{ }^{\circ} \mathrm{C}$, e foi semelhante às temperaturas encontradas nas áreas de plantio de Eucalipto, que variaram entre 20,5 e $26,2{ }^{\circ} \mathrm{C}$ (Tabela 2; Figura 3(b)). No entanto, a supressão da floresta nativa e do Eucalipto (solo exposto) aumentou a temperatura da superfície em $5,6^{\circ} \mathrm{C}$ no período de estiagem e em $3,2^{\circ} \mathrm{C}$ no período de chuva. 
Figura 3 - Albedo (a) e temperatura da superfície $\left({ }^{\circ} \mathrm{C}\right)(\mathrm{b})$ em diferentes usos do solo, nos períodos sazonais de 2015 a 2017.

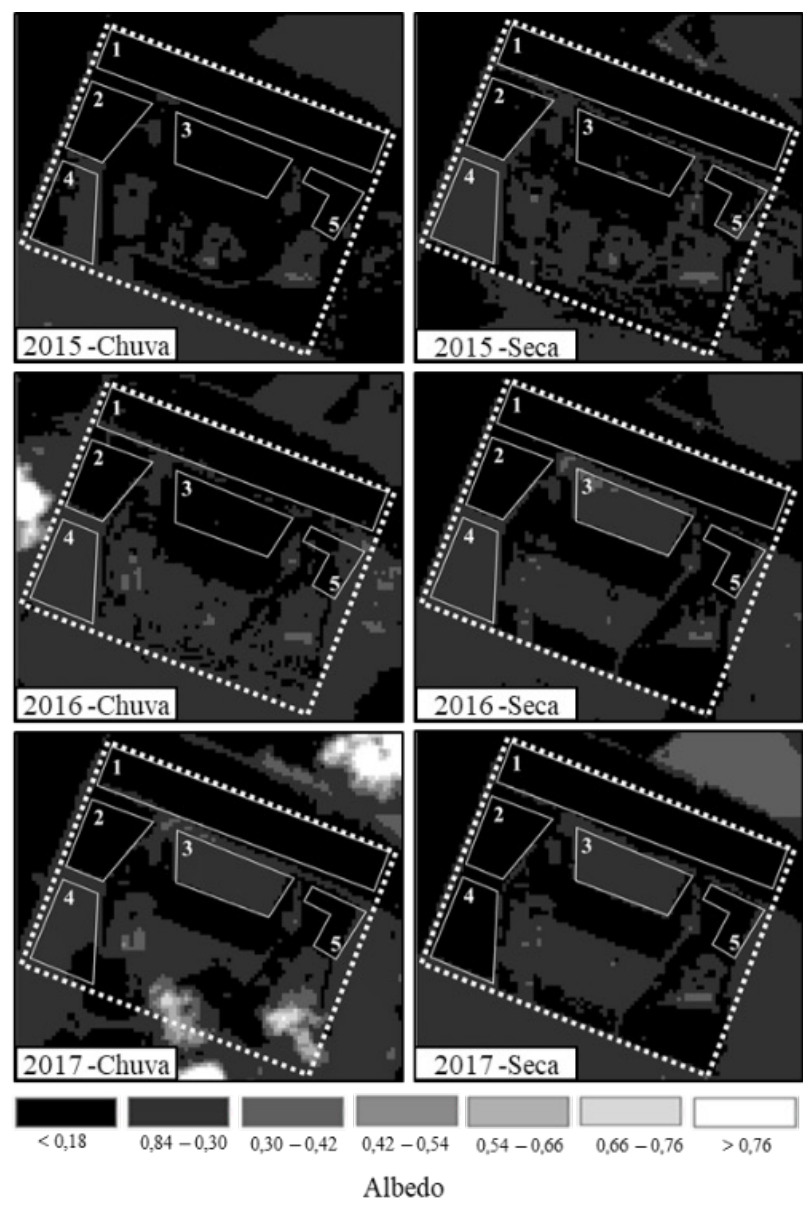

(a)

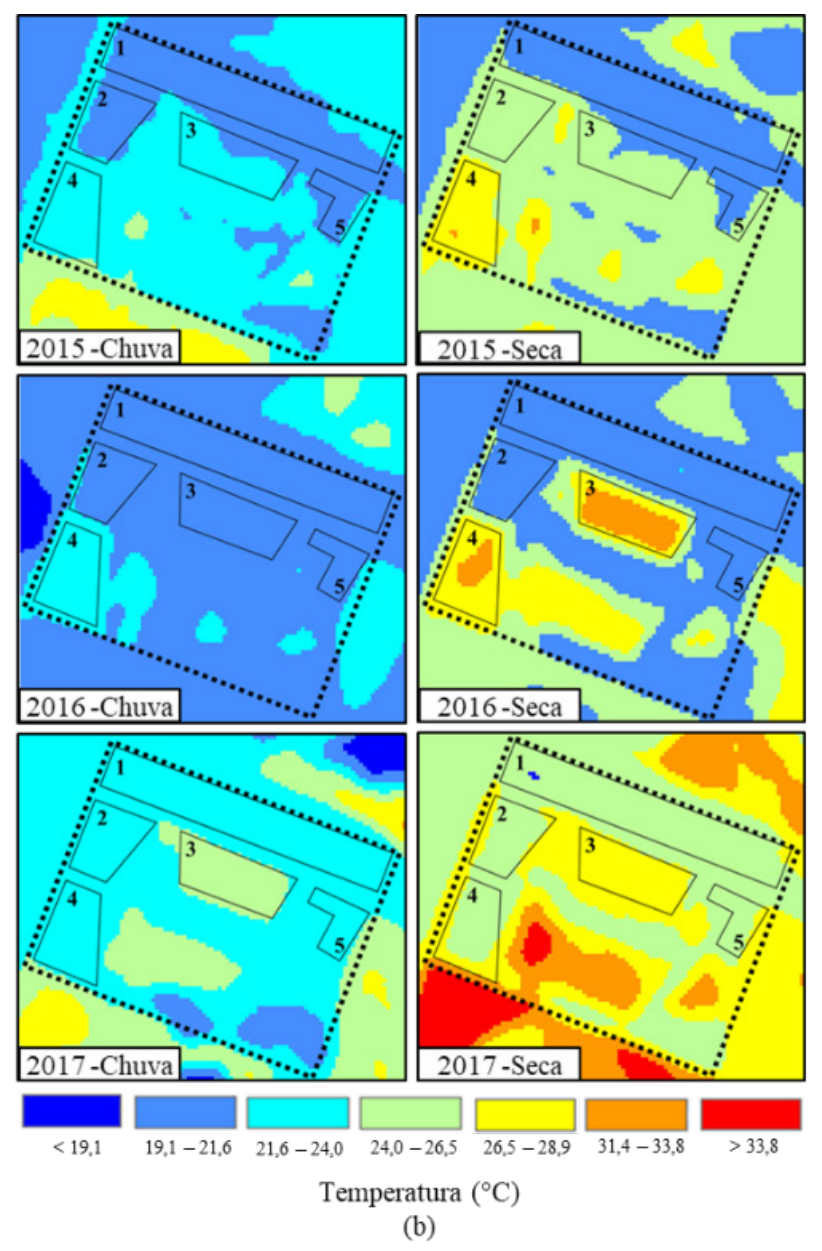

E. urophylla e E. urograndis, provavelmente devido ao semelhante albedo entre os plantios clonal e devido à uniformidade do plantio e ausência de descontinuidade da cobertura dos talhões (REIS et al., 2014). Em contrapartida, o Rn do solo exposto foi $13 \%$ (chuva) e $20 \%$ (seca) menor que na floresta nativa (Tabela 2 e Figura 4(a)). A ausência de cobertura vegetal implica na maior reflexão da radiação de ondas curtas e maior emissão de radiação de ondas longas, reduzindo o $\mathrm{Rn}$ da superfície (ANDRADE et al., 2014).

Os maiores valores de evapotranspiração da superfície (ET) foram encontrados na área de floresta nativa, variando entre 3,3 e 5,2 $\mathrm{mm} \mathrm{dia}^{-1}$, seguido pelos talhões de Eucalipto, variando entre 2,6 e 4,8 $\mathrm{mm} \mathrm{dia}^{-1}$, e da área de solo exposto, variando entre 0,7 e 2,9 $\mathrm{mm}$ $\mathrm{dia}^{-1}$ (Tabela 2; Figura 4(b)). Valores semelhantes de ET foram encontrados por Angelini et al. (2017), que encontraram valores entre $2,0 \mathrm{~mm} \mathrm{dia}^{-1}$ e $5,0 \mathrm{~mm} \mathrm{dia}{ }^{-1}$ em áreas de Cerradão e entre $1,4 \mathrm{~mm} \mathrm{dia}^{-1}$ e $4,5 \mathrm{~mm}$ $\mathrm{dia}^{-1}$ em área Cerrado Stricto Sensu, respectivamente, assim como, Facco (2004) que encontrou ET entre 2,90 $\mathrm{mm} \mathrm{dia}{ }^{-1}$ e $3,40 \mathrm{~mm} \mathrm{dia}{ }^{-1}$ em plantio de Eucalipto com dois a quatro anos de idade.

Os valores maiores de evapotranspiração na floresta nativa, mesmo no período de estiagem, são justificados $3 \%$ e $9 \%$ no período seco, respectivamente. Houve ainda pouca alteração no $\mathrm{Rn}$ entre as idades e as variedades 
Figura 4 - Saldo de Radiação diário (W m $\left.{ }^{-2} \mathrm{dia}^{-1}\right)$ (a) e evapotranspiração ( $\left.\mathrm{mm} \mathrm{dia}^{-1}\right)$ (b) em diferentes usos do solo, nos períodos sazonais de 2015 a 2017

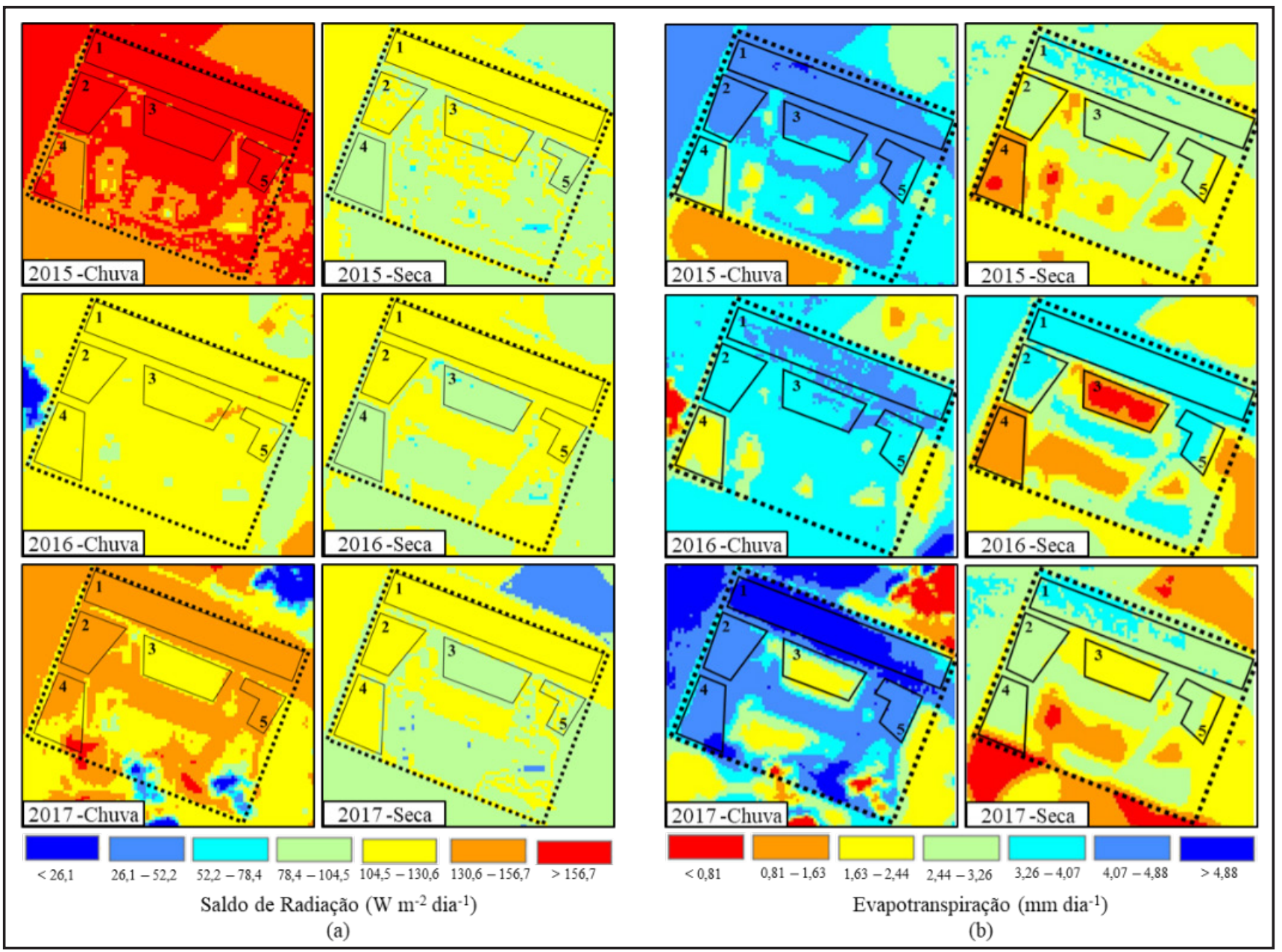

pela estrutura e composição da vegetação que apresenta espécies das fitofisionomias Cerrado e Amazônia, cuja alta rugosidade aerodinâmica, capacidade de extração de água de regiões mais profundas do solo e heterogeneidade do dossel proporciona maior troca de água no sistema planta-atmosfera (FISHER et al., 2007; BRANNSTROM et al., 2008; BETTS et al., 2008; VOURLITIS et al., 2008).

A conversão de áreas de floresta em pastagem, agricultura e solo exposto, porém, podem ser responsáveis por reduções de $60 \%$ a $70 \%$ na evapotranspiração, conforme observado por Angelini et al. (2017) e Silva et al. (2014). Esses mesmos autores, entretanto, ressaltam que essa redução pode ser minimizada para $12 \%$ quando áreas de Cerrado são comparadas com reflorestamento de Eucalipto.

Salienta-se que os resultados apresentados nesse estudo são preliminares sendo necessário ainda a comparação deles com dados obtidos in loco em estações micrometeorológica para quantificar a magnitude dos erros relativos associados à aplicação do algoritmo R-SSEB na estimativa dos parâmetros biofísicos. Contudo, conforme observado por ARAÚJO et al. (2017) que ao calibrar o modelo R-SSEB para toda a região Nordeste do Brasil não foram observadas diferenças significativas para os dados obtidos pelo modelo e os dados medidos in loco.

\section{Conclusões}

A substituição da floresta nativa por plantio de Eucalipto alterou os parâmetros biofísicos e a evapotranspiração da superfície na área de transição Cerrado-Amazônia. Os plantios de Eucalipto, independentemente da idade ou clone, obtiveram NDVI e temperatura da superfície $3 \%$ maior, assim como, saldo de radiação e evapotranspiração da superfície $4 \%$ menor ao da floresta nativa.

O desmatamento com exposição do solo aumentou o albedo e a temperatura da superfície de $69 \%$ e $14 \%$ no período chuvoso e $109 \%$ e $34 \%$ no período de estiagem e reduziu o NDVI, o saldo de radiação e evapotranspiração da superfície em $63 \%, 13 \%$ e $57 \%$ no período chuvoso e $58 \%, 20 \%$ e $26 \%$ no período seco.

\section{Agradecimentos}

A pesquisa foi apoiada pela Universidade Federal de Mato Grosso (UFMT), Programa de Pós-Graduação em Física Ambiental (PPGFA/IF/UFMT), Instituto Federal de Mato Grosso (IFMT), Coordenação de Aperfeiçoamento de Pessoal de Nível Superior (CAPES), Conselho Nacional de Desenvolvimento Científico e Tecnológico 
(CNPq, processos n 310879/2017-5 e 305761/2018-8; Edital Universal 01/2016, processo no 407463/2016-0) e Fundação de Amparo à Pesquisa do Estado de Mato Grosso (FAPEMAT - PRONEM 2014, processo nº 561397/2014).

\section{Referências}

ALLEN RG, TASUMI M, TREZZA R. Satelliite-based energy balance for mapping evapotranspiration with internalized calibration (METRIC) - Model. J Irrig Drain Eng. 2007;133:380-394.

ALVARES CA, MUNHOZ JSB, STAPE JL, STAHL J, ANDRADE L, SANTOS G, et al. Caracterização da dinâmica dos índices de vegetação NDVI e EVI em plantações de Pinus do Brasil. In: Anais XVI Simpósio Brasileiro de Sensoriamento Remoto-SBSR, Foz do Iguaçu, Brasil, p. 2866-2873.

ANDRADE R, VICTORIA DDC, NOGUEIRA S, MASSAGLI G, AGNESE M. Estimativa do albedo e do saldo de radiação à superfície utilizando imagem MODIS-Aqua na Bacia do Rio Ji-Paraná, RO. Boletim de Pesquisa e Desenvolvimento. Embrapa Monitoramento por Satélite: Campinas, 2010. 20 p.

ANDRADE AMD, MOURA MAL, SANTOS AB, CARNEIRO RG, JUNIOR RS. Radiação fotossintéticamente ativa incidente e refletida acima e abaixo do dossel de Floresta de Mata Atlântica em Coruripe, Alagoas. Rev. bras. meteorol. 2014;29(1):68-79.

ANGELINI LP, SILVA E SILVA PCB, FAUSTO MA, MACHADO NG, BIUDES MS. Balanço de Energia nas Condições de Mudanças de Uso do Solo na Região Sul do Estado de Mato Grosso. Rev. bras. meteorol. 2017;32(3):353-363.

ARAÚJO AL, SILVA MT, SILVA BB, SANTOS CAC, AMORIM MRB. Modelagem Simplificada Para Estimativa do Balanço de Energia à Superfície em Escala Regional (R-SSEB). Rev. bras. meteorol. 2017;32(3):433-446.

ARTAXO P, DIAS MAFDS, NAGY L, LUIZÃO FJ, CUNHA HBD, QUESADA CA, et al. Perspectivas de pesquisas na relação entre clima e o funcionamento da Floresta Amazônica. Cien. Cult. 2014;66(3):41-46.

ASSOCIAÇÃO BRASILEIRA DE PRODUTORES DE FLORESTAS PLANTADAS (ABRAF). Anuário estatístico da ABRAF 2013 - ano base 2012. ABRAF: Brasília, 2013. 148p.

BASTIAANSSEN WGM. SEBAL - based sensible and latent heat fluxes in the irrigated Gediz Basin, Turkey. J. Hydro. 2000;229:87-100.
BETTS R, SANDERSON M, WOODWARD S. Effects of large-scale Amazon forest degradation on climate and air quality through fluxes of carbon dioxide, water, energy, mineral dust and isoprene. Philos Trans R Soc Lond B Biol Sci Ser. B. 2008;393:1873-1880.

BIUDES MS, VOURLITIS GL, MACHADO NG, DE ARRUDA PHZ, NEVES GAR, DE ALMEIDA LOBO, F, et al. Patterns of energy exchange for tropical ecosystems across a climate gradient in Mato Grosso, Brazil. Agric. For. Meteorol. 2015;202:112-124.

BRANNSTROM C, JEPSON W, FILIPPI AM, REDO D, XU Z, GANESH S. Land change in the Brazilian Savanna (Cerrado), 1986-2002: comparative analysis and implications for land-use policy. Land use policy. 2008;25(4):579-595.

CHRISTINA M, NOUVELLON Y, LACLAU JP, STAPE JL, BOUILLET JP, LAMBAIS GR, et al. Importance of deep water uptake in tropical eucalypt forest. Functional Ecology. 2017;31(2):509-519.

CLAVERIE M, VERMOTE EF, FRANCH B, MASEK JG. Evaluation of the Landsat-5 TM and Landsat-7 ETM + surface reflectance products. Remote Sens Environ. 2015;169:390-403.

CRISTIANO PM, MADANES N, CAMPANELLO PI, DI FRANCESCANTONIO D, RODRÍGUEZ SA, ZHANG YJ, et al. High NDVI and potential canopy photosynthesis of South American subtropical forests despite seasonal changes in leaf area index and air temperature. Forests. 2014;5(2)287-308.

CUNHA JEBL, RUFINO LAA, BERNADO BS, CHAVES LB. Dinâmica da cobertura vegetal para a Bacia de São João do Rio do Peixe, PB, utilizando-se sensoriamento remoto. Agriambi. 2012;16(5):539-548.

DOMINGUES MS, BERMANN C. O arco de desflorestamento na Amazônia: da pecuária à soja. Ambiente Soc. 2012;15(2):1-22.

DOUGHTY CE, METCALFE DB, GIRARDIN CA, AMEZQUITA FF, DURAND L, HUARACA HUASCO W, et al. Source and sink carbon dynamics and carbon allocation in the Amazon basin. Global Biogeochemical Cycles. 2015;29(5):645-655.

FACCO AG. Modelagem e simulação geoespacial dos componentes do balanço hídrico para plantios de eucaliptos em áreas de relevo ondulado. [Dissertação]. Viçosa: UFV; 2004. 87 p.

FEDERAÇÃO DA AGRICULTURA E PECUÁRIA DO ESTADO DE MATO GROSSO (FAMATO). Diagnóstico de Florestas Plantadas do Estado de Mato Grosso. Cuiabá: IMEA; 2013. 106 p.

FERREIRA JCV. Mato Grosso e seus municípios. Cuiabá: Secretaria de Estado de Educação, 2001. 
FISHER RA, WILLIAMS M, COSTA D, LOLAA, MALHI Y, DA COSTA RF, et al. The response of an Eastern Amazonian rain forest to drought stress: results and modelling analyses from a throughfall exclusion experiment. Glob. Change Biol. 2007;13(11):2361-2378.

FU B, BURGHER I. Riparian vegetation NDVI dynamics and its relationship with climate, surface water and groundwater. J. Arid Environ. 2015;113:59-68.

GODOY GOERGEN LC, DE VARGAS KILCA R, DA SILVA NARVAES I, SILVA MN, SILVA EA, PEREIRA RS, et al. Distinção de espécies de eucalipto de diferentes idades por meio de imagens TM/Landsat 5. Pesq. agropec. bras. 2016;51(1):53-60.

HUETE A, DIDAN K, MIURA T, RODRIGUEZ EP, GAO X, FERREIRA LG. Overview of the radiometric and biophysical performance of the MODIS vegetation indices. Remote Sens Environ. 2002;83:195-213.

LACLAU JP, SILVA EAD, RODRIGUES LAMBAIS G, BERNOUX M, LE MAIRE G, STAPE JL, et al. Dynamics of soil exploration by fine roots down to a depth of 10 $m$ throughout the entire rotation in Eucalyptus grandis plantations. Frontiers in plant science. $2013 ; 4: 243$.

LEWINSOHN TM, PRADO PI. Biodiversidade Brasileira: síntese do estado atual do conhecimento. São Paulo: Editora Contexto; 2002. $176 \mathrm{p}$.

LLOYD J, FARQUHAR GD. Effects of rising temperatures and $\mathrm{CO}_{2}$ on the physiology of tropical forest trees. Philos. Trans. R. Soc. Ser. B. 2008;363:1811-1817.

MARQUES HO, BIUDES MS, PAVÃO VM, MACHADO NG, QUERINO CAS, DANELICHEN VHM. Estimated net radiation in an Amazon-Cerrado transition forest by Landsat 5 TM. J Appl Remote Sens. 2017;11(4):046020.

MARTINS AL, ROCHA DA CUNHA C, RODRIGUES PEREIRA VM, DE MORAIS DANELICHEN VH, GOMES MACHADO N, DE ALMEIDA LOBO F, et al. Mudanças em índices biofísicos devido à alteração da cobertura do solo em área nativa de Cerrado em Mato Grosso. Ciência e Natura. 2015;37(4):152-159.

OLIVEIRA T, CARVALHO L, OLIVEIRA L, LACERDA W, ACERBI F. NDVI Time Series for Mapping Phonological Variability of Forests Across the Cerrado Biome in Minas Gerais, Brazil. In: Zhang X. Phenology and Climate Change In Tech: Shanghai; 2012. p. 253-272.

PAVÃO VM, QUERINO CAS, QUERINO JKAS, MARTINS PAS, SIQUEIRA LF, LEITE LO, et al. Análise da radiação solar global (rg) e albedo superficial sobre um ecossistema de floresta nativa em Humaitá - AM durante o período chuvoso. Revista EDUCAmazônia. 2014;12(1):42-56.
PAVÃO VM, QUERINO C, BENEDITTI C, PAVÃO LL, QUERINO J, MACHADO, NG, et al. Temperatura e albedo da superfície por imagens TM Landsat 5 em diferentes usos do solo no sudoeste da Amazônia brasileira. RBCLima. 2015;16:169-183.

PAVÃO VM, NASSARDEN DCS, PAVÃO LL, MACHADO NG, BIUDES MS. Impacto da Conversão da Cobertura Natural em Pastagem e Área Urbana sobre Variáveis Biofísicas no Sul do Amazonas. Rev. bras. meteorol. 2017;32(3):343-351.

PEREIRA G, MORAES EC, ARAI E, OLIVEIRA LGL. Estudo preliminar da estimativa da reflectância e albedo de microssistemas pantaneiros a partir de imagens de satélite. Revista Brasileira de Cartografia. 2007;59(1):55-61.

PONZONI FJ. Comportamento Espectral da Vegetação. In: Meneses PR, Netto JSM. Sensoriamento remoto, reflectância dos alvos naturais. Brasília: Editora Universidade de Brasília, Embrapa Cerrados; 2001. p 157-199.

PONZONI FJ, PACHECO LRF, DOS SANTOS SB, ANDRADES FILHO CO. Caracterização espectrotemporal de dosséis de Eucalyptus spp. mediante dados radiométricos TM/Landsat5. Cerne. 2015;21:267275.

QUERINO CAS, BENEDITTI CA, MACHADO NG, DA SILVA MJG, QUERINO JKAS, DOS SANTOS NETO LA, et al. Spatiotemporal NDVI, LAI, albedo, and surface temperature dynamics in the southwest of the Brazilian Amazon forest. J Appl Remote Sens. 2016;10(2):026007.

REIS MG, RIBEIRO A, BAESSO RCE, SOUZA WG, FONSECA S, LOOS RA. Balanço hídrico e de energia para plantios de eucalipto com cobertura parcial do solo. Ci. F1. 2014;24(1):117-126.

ROUSE JW, HAAS RH, SCHELL JA, DEERING DW. Monitoring vegetation systems in the great plains with ERTS. In: Earth Resources Technology Satellite-1 Symposium, 3. Washington, 1974. Proceedings. Washington: NASA. Goddart Space Flight Center, 1974. p. 309-317.

SALAZAR A, BALDI G, HIROTA M, SYKTUS J, McALPINE C. Land use and land cover change impacts on the regional climate of non-Amazonian South America: A review. Glob. Planet. Change. 2015;128:103-119.

SCHUURMANS JM, TROCH PA, VELDHUIZEN AA, BASTIAANSEEN WGM, BIERKENS MFP. Assimilation of remotely sensed latent heat flux in a distributed hydrological model. Adv Water Resour. 2003;26:151-159.

SENAY GB, BUDDE M, VERDIN JP, MELESSE AMA. Coupled remote sensing and simplified surface energy balance approach to estimate actual evapotranspiration from irrigated fields. Sensors. 2007;7:979-1000. 
SENAY GB, BOHMS S, SINGH RK, GOWDA PH, VELPURI NM, ALEMU H, et al. Operational evapotranspiration mapping using remote sensing and weather datasets: A new parameterization for the SSEB approach. J Am Water Resour Assoc. 2013;49(3):577-591.

SHARKEY TD. Effects of moderate heat stress on photosynthesis: Importance of thylakoid reactions, rubisco deactivation, reactive oxygen species, and thermotolerance provided by isoprene. Plant Cell Environ. 2005;28:269-277.

SILVA SALLO F, DE FRANÇA MS, MARTINS DE MORAIS D, RODRIGUES R, SACARDI BIUDES M. Estimativa de componentes do balanço de radiação em diferentes tipos de uso e cobertura do solo. Ambiagua. 2014;9(2):347- 358 .

SILVA BB, WILCOX BP, SILVA VDPR, MONTENEGRO SMGL, OLIVEIRA LMM. Changes to the energy budget and evapotranspiration following conversion of tropical savannas to agricultural lands in São Paulo State, Brazil. Ecohydrology. 2015;8(7):1272-1283.

SOUZA AP, MOTA LL, ZAMADEI T, MARTIN CC, ALMEIDA FT, PAULINO J. Classificação climática e balanço hídrico climatológico no estado de Mato Grosso. Nativa. 2013;1(1):34-43.

TANAKA AA, DE SOUZA AP, KLAR AE, DA SILVA AC, GOMES AWA. Evapotranspiração de referência estimada por modelos simplificados para o Estado do Mato Grosso. Pesq. agropec. bras. 2016;51(2):91-104.

TASUMI M, ALLEN RG, TREZZA R. At-surface reflectance and albedo from satellite for operational calculation of land surface energy balance. J Hydrol Eng. 2008;13(2):51-63.

TRENTIN AB, TRENTIN CB, SALDANHA DL, KUPLICH TM. Modis time series analysis and TRMM in Eucalyptus plantations. Mercator, 2018;17:1:11.

VOURLITIS GL, DE SOUZA NOGUEIRA J, DE ALMEIDA LOBO F, SENDALL KM, DE PAULO SR, ANTUNES DIAS CA, PINTO OB, DE ANDRADE NLR. Energy balance and canopy conductance of a tropical semi-deciduous forest of the southern Amazon Basin. Water Resour. Res. 2008:44(3):1-14.

VOURLITIS GL, DE SOUZA NOGUEIRA J, DE ALMEIDA LOBO F, PINTO JUNIOR OB. Variations in evapotranspiration and climate for an Amazonian semi-deciduous forest over seasonal, annual, and El Niño cycles. Int J Biometeorol. 2015;59(2):217-230. 